\title{
NANOEMULSIONS FOR PROSTRATE CANCER THERAPY: AN OVERVIEW
}

\author{
MANIKANDAN P1*, SUNDARA GANAPATHY R ${ }^{2}$
}

${ }^{1}$ Research scholar, Karpagam University, Karpagam Academy of Higher Education, Coimbatore, Tamil Nadu, India. ${ }^{2}$ Faculty of Pharmacy, Karpagam University, Karpagam Academy of Higher Education, Coimbatore, Tamil Nadu, India. Email: psmanikandan12@gmail.com

Received: 27 January 2017, Revised and Accepted: 16 February 2017

ABSTRACT

The objective of this review is to focus the inferences of low/poor bioavailability and lack of dose proportionality for the oral delivery of drugs in prostate cancer therapy. To overcome such problems, various formulation strategies has been reported including various methods for the use of surfactants, cyclodextrins, solid dispersions, micronization, permeation enhancers, and lipids. Flutamide is an antiandrogen drug and used for the therapy of prostate cancer. The flutamide drug is having limited clinical application due to its poor water solubility and needs enhancement of its dissolution rate in simulated gastric fluids. The lipid-based formulations such as nanoemulsion have been shown to improve the solubility and oral absorption of lipophilic drugs. To conclude, this article emphasizes the various approaches of nanoemulsion based formulation for prostate cancer therapy.

Keywords: Nanoemulsion, Prostate cancer, Flutamide, Antiandrogen drug, Lipophilic drugs.

(c) 2017 The Authors. Published by Innovare Academic Sciences Pvt Ltd. This is an open access article under the CC BY license (http://creativecommons. org/licenses/by/4. 0/) DOI: http://dx.doi.org/10.22159/ajpcr.2017.v10i5.17307

\section{INTRODUCTION}

Cancer is the global risk factor in the current scenario. Mostly cancer risks were reported to be due to environmental hazards majorly due to air pollutants associated with vehicle smoke, smoke exposure by the non-smokers, ultraviolet radiations from sunlight, radon gas exposure, asbestos exposure, diesel exhaust pollutants, high arsenic content water consumption, and chemicals exposure. The treatment options such as surgery, radiation therapy, and chemotherapy were commonly practiced for cancer. In this regimen, the current treatment options under research includes targeted therapy (with targeting ligands focusing specific diseased receptors), immunotherapy, magnetitebased delivery therapy, light-based therapy (photodynamic therapy), ultra sound-based therapy (sonodynamic therapy) using conventional nanoparticulate systems such as nanoparticles, nanoemulsions, nanodispersions, nanosuspensions, nanomicelles, and siRNA coupled combination nanoformulations were utilized. Since there are various approaches for the cancer treatments, but the success level is not satisfactory, requires retreatments [1]

The transparent/translucent multi-phase colloidal systems composed of two immiscible phases (dispersed internal phase (droplets) and the other is the continuous external phase) which are only kinetically stable and used for the treatment of various ailments were termed as nanoemulsions. Nanoemulsions range approximately between 10 and $500 \mathrm{~nm}$ either in oil/water and water/oil forms. Natural, semi-synthetic, and synthetic oils were used for the formation of nanoemulsions. In view of targeted approach currently biodegradable polymeric nanoparticles, hydrogels, micelles, liposomes, microemulsions, nanoemulsions, dendrimers, nanoshells, nanotubes, nanomaterials, polymersomes, nucleic-acid-based nanoparticles, magnetic nanoparticles, polynucleotide nanoparticles, and virus nanoparticles were developed and utilized for targeting drugs to the target site of cancerous organs. The major components of nanoemulsions include oil, surfactant, and cosurfactant. The efficiency of nanoemulsions rely on the smaller size of droplets, the larger surface area, the low surface tension of the entire system, and the low interfacial tension of the droplets [2].

Whereas, microemulsions a new pharmaceutical interest for drug delivery are thermodynamically stable low viscous isotropic homogeneous system [3] that can be prepared over a wide range of surfactant concentrations in which two immiscible liquids are mixed to form a single phase by appropriate surfactant which serves as a promising vehicle possessing powerful ability to deliver drugs through the skin. Advantages of microemulsions over coarse emulsion include spontaneous formation, transparent, enhanced permeation through biologic membranes, increased bioavailability and less inter- and intraindividual variability in drug pharmacokinetics [4]. Several factors affect the transdermal drug delivery of microemulsions include the affinity of a drug to the internal Phase I microemulsion, ingredients reducing the barrier of the stratum corneum, increased concentration gradient toward skin and the dispersed phase acting as a reservoir. In transdermal delivery, the goal of dosage design is to maximize the flux through the skin into systemic circulation. A useful strategy for improving percutaneous flux is to improve the concentration of drug or by choosing an appropriate vehicle for delivery [5]. However when compared to microemulsions, the nanoemulsions were playing a vital role for cancer drug delivery recently.

\section{NOVEL PREPARATION METHODS FOR NANOEMULSIONS}

To reflect an efficient treatment drugs/bioactive material/bioactive proteins may be formulated into nanoemulsions, provided which may elicit good bioavailability and enhanced therapeutic response. To prepare a stable nanoemulsion proper selection of appropriate composition, controlled addition of the components, and application of the shear to effectively rupture the droplets are an essential criteria [6]. The preparation methods for nanoemulsions includes highpressure homogenization (high-pressure homogenizer, at a pressure of 50-100 $\mathrm{MPa}$ ) [7], microfluidization, ultrasonication (ultrasound generators), phase inversion method, spontaneous emulsification, solvent evaporation technique, and hydrogel method. Among these ultrasonic emulsification technique also reduces the droplet size to a considerable level, prepared using biodegradable polymers, mostly applicable for hydrophobic drugs. Recently, cyclooxgenase- 2 inhibiting perfluoropoly (ethylene glycol) ether theranostic nanoemulsions has been reported by Janjic et al., 2013 to improve the aqueous solubility of celecoxib, to attain an enhanced tumor inhibiting effect using cremophor EL and PluronicP105 [8]. Fig. 1 summarizes the different novel preparation methods for nanoemulsion. 


\section{COMPONENTS OF NANOEMULSION}

Their long term physical stability makes them unique and referred as approaching chemical stability. The high colloidal nature of the nanoemulsion can be studied from a consideration of their stearic stabilization and how this is affected by the ratio of absorbed layer thickness to droplet radius [9]. An emulsion system is a system which is, of which one is the dispersed internal phase (droplets) and the other is the continuous external phase. Normally, they are in oil-in-water and water-in-oil forms, in which the core may be either oil or water, respectively. Nanoemulsions are normally prepared using surfactants that are generally considered safe for human consumption by the Food and Drug Administration. The type of surfactant and their concentration in the aqueous phase are chosen to provide better stability against coalescence. Several types of oils (natural, semi-synthetic, and synthetic) are used in the formulations of nanoemulsions. The ability of nanoemulsions to dissolve a large amount of poorly soluble drugs along with their mutual compatibility and capacity to protect the drugs from hydrolysis and enzymatic degradation makes them an ideal vehicle for drug delivery [10]. Fig. 2 pictures the basic components of a nanoemulsion.

\section{OPTIMIZATION OF NANOEMULSIONS FORMATION CAPACITY}

To optimize nanoemulsions, the nanoemulsions formation, the pseudoternary phase diagrams comprising oil, surfactants-cosurfactants mixture (smix) and double distilled water were utilized using the aqueous titration method. Phase diagrams prepared by varying weight ratios of surfactant to cosurfactants were used. The ratios were selected based on the concentration of surfactants/cosurfactants. For each diagram at a particular smix ratio, the ratios of oil to smix are varied.

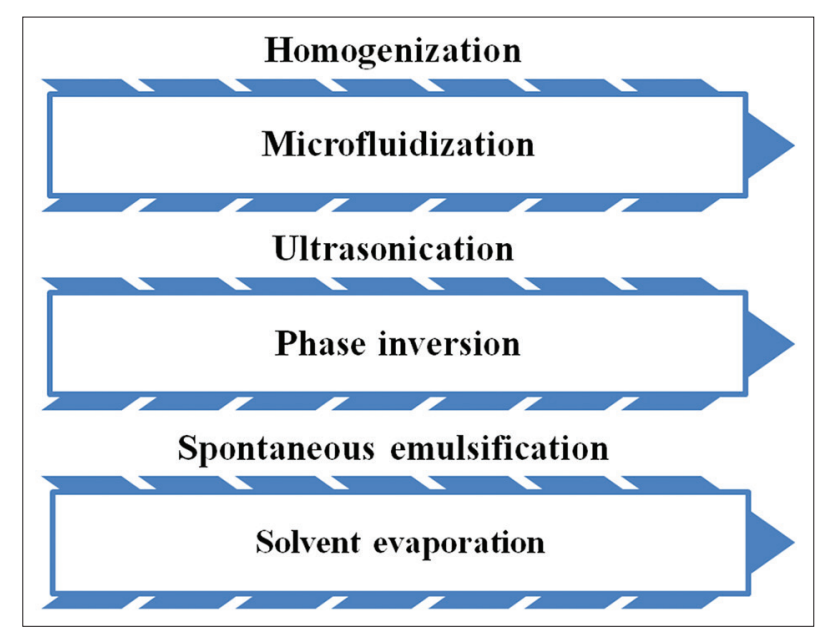

Fig. 1: Novel preparation methods for nanoemulsion

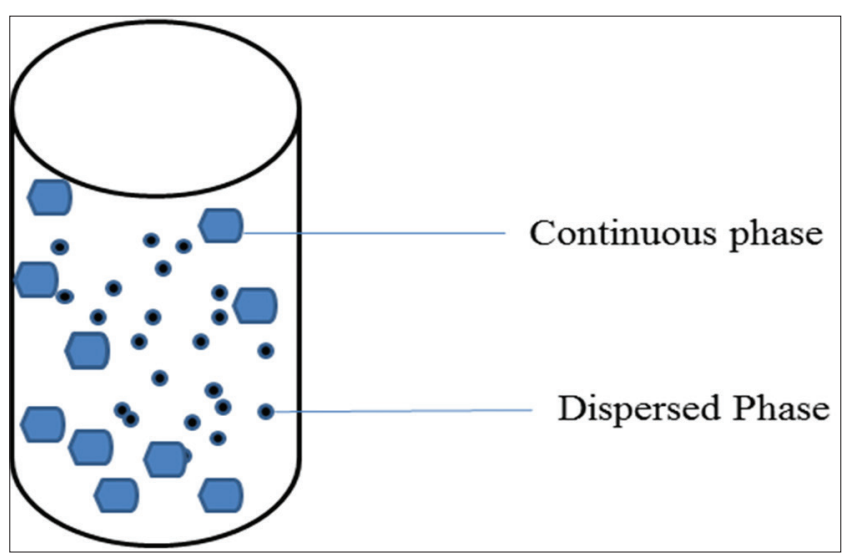

Fig. 2: Basic components of nanoemulsions
The mixture of oil and smix at particular ratios are diluted with water drop-wise, under magnetic stirring. The nanoemulsions formation capacity were marked on phase diagram with one axis representing the aqueous phases, other the oily phase and the next one representing the smix at a particular weight ratio [10].

\section{PHARMACEUTICAL APPLICATIONS OF NANOEMULSIONS}

In this regimen, the common pharmaceutical applications of nanoemulsions were they were considered as a potent vehicle for delivery of cosmetics upon skin layers due to its lipophilicity. Antimicrobial nanoemulsions composed of oil, water, surfactants, and alcohol were used against bacteria), viruses, fungi and spores. Nanoemulsion will get fused with lipid-containing organisms and it is enhanced by the electrostatic attraction between emulsion opposite charges. As a vehicle for the proteins delivery nanoemulsions possesses an active role against inactive organisms to the mucosal surfaces to produce an immune response. Nanoemulsions are used in the field of cell culture for the delivery of various substances to the culture medium with the major advantages of transparency; nanosized droplets possess the high rate of uptake and cellular bioavailability. As a major role in parental drug delivery nanoemulsions serves as an ideal vehicle for the transport of drugs because of their ability to dissolve the hydrophobic substances and protection from hydrolysis and enzymatic degradation. As common phenomena both microemulsions and nanoemulsions have their specific applications. Chen et al. 2014 developed Ganoderma lucidum triterpene loaded microemulsion using Coix lacrymajobi seed as oil phase for anti-tumor effect. The characterized the developed microemulsions for its size, shape, and morphology. The drug loading effect of the developed formulation was checked by UV spectrophotometer at $584 \mathrm{~nm}$ with an encapsulation of around $80.0-84.0 \%$. The anti-proliferative effect of the developed microemulsion was checked in A549 cells and found the half maximal cytotoxic inhibitory concentration was $0.62 \mathrm{mg} / \mathrm{ml}$ of the crude extract. The results of their studies concludes that microemulsion based formulations can improve the solubility of triterpenes [11]. Han et al. (2009) developed novel 10-methoxy-9-nitrocamptothecin (MONCPT) nanoemulsion (o/w type) to enhance its solubility, stability, and antitumoractivity. Development of nanoemulsion was achieved using lipoid E80 and cremophor EL as main emulsifiers by microfluidization method. Solubility and internalization of lactone form of MONCPT in nanoemulsion in the interfacial surfactant layer of the nanoemulsion was 200-fold higher than in water. MONCPT nanoemulsion shows no obvious hemolysis in rabbit erythrocytes. MONCPT nanoemulsion showed increased cytotoxicity with the suppression rate of is $93.6 \%$, and only $24.2 \%$ was observed for MONCPT injection at the same dosage. In vivo imaging of tumor in S180-bearing mice evident that more accumulation in tumor cells [12].

\section{FLUTAMIDE}

Flutamide(2-methyl-N-(4-nitro-3-(trifluoro methyl) phenylpropanamide) is a non-steroidal antiandrogenicpoorly water soluble drug competes with testosterone and its powerful metabolite, dihydro-testosterone binds to androgen receptors in the prostate gland. Flutamide possess the capacity to induce the androgen levels in womens, especially those with polycystic ovarian syndrome. Flutamide is practically insoluble in water $(9.45 \mathrm{mg} / \mathrm{L})$ and possess higher hydrophobicity (log P 2.6). Flutamide holds poor absorption, dissolution rate, and bioavailability due to its poor wettability, low aqueous solubility, poor permeability, rapid first pass hepatic metabolism, and low concentration at the absorption surface. Lipid-based drug delivery systems (enhancing membrane permeability and lymphatic transport) may improve the solubility/bioavailability of flutamide. Developing novel formulations may improve the solubility, dissolution profile, and cellular uptake/target ability at the diseased sites. The common side effects of flutamide include hepatotoxicity, nausea, and diarrhea. Flutamide gets metabolized into 2-hydroxyflutamide, which also possesses anti-androgenic properties [13,14]. Table 1 represents flutamide loaded nanoformulations and its biological applications [15-20]. 
Table 1: Flutamide loaded nanoformulations and its biological applications

\begin{tabular}{|c|c|c|c|c|}
\hline S. No & Nano products & Polymers utilized & Biological applications & References \\
\hline 1. & Hydrogels & Poly-4-acrylolylmorpholine acrylic acid & Anti-cancer effect & [15] \\
\hline 2. & Lyophilized dispersions & B-cyclodextrin/hydroxypropyl- $\beta$-cyclodextrin & Prostate cancer treatment & [16] \\
\hline 3. & Nanoparticles & Ionically crosslinked casein & Anti-cancer effect & [17] \\
\hline 4. & Self nanoemulsfying drug delivery system & Sesame oil, Tween 20, Poly ethylene glycol 400 & Anti-cancer effect & [18] \\
\hline 5. & Sustained-release matrix tablets & $\begin{array}{l}\text { Cellulose ethers, natural gums and compressible } \\
\text { eudragits }\end{array}$ & Anti-cancer effect & [19] \\
\hline 6. & Orodispersible tablets & Sodium starch glycolate & Prostate cancer treatment & {$[20]$} \\
\hline
\end{tabular}

\section{PROSTRATE CANCER}

Prostate cancer is a type of cancer which affects the male reproductive system with cancerous tissues in the prostate, with altered shape of the prostate gland holds the highest incidence among all forms of cancers and is the second most deadly cancer in men, even in the US also it has been reported to be the second leading cause for cancer-related deaths in mens, after lung cancer [21]. The incidence of prostate cancer is high at Northern America, Australia, New Zealand, Northern and Western Europe. In India also the incidence rate of prostate cancer is at the highrisk level [22]. Prostate cancer was basically dependent on androgen signaling pathway receptor. It is a basic protein composed of 919 repeated aminoacids sequences such as poly-glutamine, poly-glycine, and poly-prolinerepeats of variable lengths. These androgen receptor has three major domains such as amino-terminal transcriptional activation domain, a DNA-binding domain containing 2 zinc finger motifs, and a carboxyl terminal ligand-binding domain which regulates the switch by which androgens control the transcriptional activity of the receptor. The pathogenesis of prostate cancer greatly depends on the alterations in zinc accumulation, alteration of metabolism, and citrate production. Altered proteins in the androgen receptor signaling leads to altered cellular conditions which may also play an important role in prostate cancer pathogenesis. Src signaling based pathway also induces androgen-induced prostate cancer cells proliferation. The PI3K/PTEN/Akt/mTOR pathway mediates cell survival and neoplastic transformation is also constitutes in the development of prostate cancer cells, mainly due to the tumor suppressor gene [23]. Moreover, prostate cancer cells produce prostatic acid phosphatase, which acts on bone remodeling. The pathophysiology indicates that inhibiting androgen receptor may be suitable choice to target the prostate cancer cells. Zinc is the major contributor for prostate cancer cells progression [24]. A study showed that prostate cancer is influenced by genetic and several non-genetic factors. The genetic factors include genome-wide linkage and association studies have been used to identify genomic loci contributing to prostate cancer susceptibility. The non-genetic factors are age, family history and obesity [25]. Diet which increases the bioavailability of zinc may also the risk factor for prostate cancer individuals. The treatment options adopted for prostate cancer includes surgery, chemotherapy, radiation therapy, and hormonal therapy. Whereas these treatment approaches were not promising and they require additional repeated treatments, even the cancer regrowth may occur. Therefore treatment options addressing these issues may be advantageous in the current scenario.

\section{CURRENT TREATMENT APPROACHES FOR PROSTATE CANCER}

Current research trend was focusing various treatment approaches for prostate cancer. These treatment modules include various nanoparticulate drug delivery systems even coupled with or without magnetite or siRNA-based systems. Curcumin (a natural polyphenol) due to its multi-acting mechanisms at various sites such as nuclear factor-kappa B, transcription factor activator protein-1, mitogenactivated protein kinase, tumor protein 53 , nuclear $\beta$-catenin signaling, and AKT signaling pathways has been formulated into nanoparticles using cellulose has been reported for the treatment of prostate cancer. The cellulose-based curcumin nanoparticles showed the enhanced apoptotic effect and utmost cellular uptake mechanism in human prostate cancer cell lines (C4-2). These reveal the superior anti-cancer effect for the cellulose based curcumin nanoparticles [26]. Dash et al. developed multifunctional nanoparticles containing cyclopamine and paclitaxel for the treatment of prostate cancer. They entrapped both drugs in entrapped in glyceryl mono oleate-chitosan solid lipid as well as in poly(glycolic-lactic) acid polymer. The particle size of drugloaded glyceryl mono oleate-chitosan nanoparticles was found to be $278.4 \pm 16.4 \mathrm{~nm}$ with a positive zeta potential. Whereas, the particle size of drug loaded poly(lactic-co-glycolic) acid nanoparticles was found to be $234.5 \pm 6.8 \mathrm{~nm}$ in size with a negative zeta potential. The results of their studies confirm that the types of polymer used for the nanoparticles preparation plays a vital role and affected the in vitro release, cytotoxicity, and uptake of nanoparticles in the all the cell lines studied at in vitro level [27]. Thermotherapy using magnetic nanoparticles, RGD peptide for the targeted therapy, light based photodynamic therapy approach, sonodynamic therapy, photothermal destruction using near infra-red light, and magneto oscillatory therapy were the current updates for these types of cancer-based treatment approaches.

\section{FLUTAMIDE LOADED NANOEMULSION}

Flutamide which is a lipophilic drug can be formulated into nanoemulsions form using suitable oils, surfactant, and cosurfactant. Thereby the problems of poor bioavailability at the required diseased site and poor water solubilising capacity can be enhanced. The nonionic surfactants which can be used for these formulation approach may be selected as poloxamers, polysorbates, spans, vitamin E TPGS, solutol HS and polyethoxylated castor oil derivatives. Mono-, di-, and tri-glycerides can be selected as oily phase for the nanoemulsion formulation. The flutamide loaded nanoemulsions can be formulated using polyethylene glycol (using various grades) or grades selected based on the conceptual requirements. The clarity of the formulation may be assessed. The formulated nanoemulsions can be characterized by various in vitro studies (morphology, droplet size) analysis. The behavior of the nanoparticles at the cellular level (prostate cancer) based cell lines can be assessed. Finally, the bioavailability and anticancer effects of flutamide loaded nanoemulsions can be checked using prostate cancer induced animal models. Thus, this type of nano-based system which encapsulates flutamide can be the suitable system for the treatment of prostate cancer in the near future [28].

\section{TRANSDERMAL NANOEMULSION}

Formulations of nano-emulsion for administering protein vaccine with artin-M adjuvant were developed. The composition of the formulation was $3 \%$ virgin coconut oil, $16 \%$ Tween $80,8 \%$ polyethylene glycol (PEG) 400 and deionized water. Bovine serum albumin (BSA) and artin-M were incorporated in the oil phase as co-lyophilized solid dispersion in PEG 20000 with the optimum ratio of 1:1. The results of nano-emulsion formulation indicated the uniform globule diameter formation. Both BSA and artin-M were dispersed uniformly in the oil phase during the storage with no-aggregation observed in the reconstituted nanoemulsion. These studies showed that formulation of nano-emulsion could be used for spontaneous delivery of protein vaccine [29].

\section{CURCUMIN NANOEMULSION}

The study showed that encapsulation of curcumin in a nano-emulsion system is expected to increase the bioavailability of the curcumin after 
oral administration. In this study, formulation of tablet containing curcumin nanoemulsion for oral delivery was evaluated. The study results indicated that curcumin nanoemulsion could able to be formulated into solid dosage form, with good physical characteristic of the tablets [30].

\section{CONCLUSION}

Nano-emulsion formulations may holds superior effect (anti-cancer) for the treatment of prostate cancer with small size, enhanced cytotoxicity, solubilizing the drug, and target ability. It may enhance the bioavailability of the drugs at the maximal level when compared to conventional dosage forms. Currently, technologies are available for targeting the diseased site using multi-functional nano-based systems to avoid the toxicity to the healthy cells when compared to the diseased cells.

\section{REFERENCES}

1. Mason TG, Wilking JN, Meleson K, Chang CB, Graves SM. Nanoemulsions: Formation, structure and physical properties. J Phys Condens Matter 2006;18:R635-66

2. Solans C, Izquierdo P, Nolla J, Azemar N, Garcia-Celma MJ. Nanoemulsions. Curr Opin Colloid Interface Sci 2005;10:102-10.

3. Maria CC, Rubiana MM, Maria PD. Development and validation of HPLC method for the analysis of dexamethasone in microemulsions. Braz J Pharm Sci 2009;45:87-92.

4. Derle DV, Sagar BS, Rohini P. Microemulsion as a vehicle for transdermal permeation ofnimesulide. Indian J Pharm Sci 2006;68:622-5.

5. Huabing C, Xueling C, Ting W, Xiaozhi Z, Zhonghong G, Yajiang Y. A study of microemulsion systems for transdermal delivery of triptolide. J Control Release 2004;98:427-36.

6. Sukanya G, Mantray S. Review on nanoemulsions. Int J Innov Pharm Sci Res 2013;21(2):192-205.

7. Gupta PK, Pandit JK. Pharmaceutical nano technology novel nanoemulsion-high energy emulsification preparation, evaluation and application. Pharm Res 2010;3:117-38.

8. Patel SK, Zhang Y, Pollock JA, Janjic JM. Cyclooxgenase-2 inhibiting perfluoropoly (Ethylene glycol) ether theranostic nanoemulsions - in vitro study. PLoS One 2013;8(2):e55802.

9. Rajalakshmi R, Mahesh K. Critical review on nanoemulsions. Int J Innov Drug Discov 2011;1(1):1-8

10. Lovely C, Attama AA. Current state of nanoemulsionsin drug delivery. J Biomater Nano Biotechnol 2011;2:626-39.

11. Chen H, Khemtong C, Yang X, Chang X, Gao J. Nanonization strategies for poorly water-soluble drugs. Drug Discov Today 2011;16:354-60.

12. Han $\mathrm{M}$, He CX, Fang QL, Yang XC, Diao YY, Xu DH, et al. A novel camptothecin derivative incorporated in nanocarrier induced distinguished improvement in solubility, stability and anti-tumor activity both in vitro and in vivo. Pharm Res 2009;26(4):926-35.
13. Posti J, Katila K, Kostiainen T. Dissolution rate limited bioavailability of flutamide and in vitro-in vivo correlation. Eur J Pharm Biopharm 2000;49:35-9

14. Verma A, Singh MK, Kumar B. Development and characterization of flutamide containing self-micro emulsifying drug delivery system. Int J Pharm Pharm Sci 2011;3(4):60-5.

15. Abou-Taleb MF, Signey SE, Kemary ME. Sustained release flutamide from radiation cross linked poly- 4- acryl- morpholine acrylic acid hydrogels. Macromol Res 2012;20(4):407-14

16. Elgindy N, Elkhodairy K, Molokhia A, Elzoghby A. Lyophilization monophase solution technique for improvement of the physicochemical properties of an anticancer drug flutamide. Eur J Pharm Biopharm 2010;74:397-405.

17. Elzoghby AO, Helmy MW, Samy WM, Elgindy NA. Novel ionicallycrosslinked casein nanoparticles for flutamide delivery: Formulation, characterization and in vivo pharmacokinetics. Int $\mathrm{J}$ Nanomed 2013;8:1721-32

18. Jeevanajyothi B, Sreelakshmi K. Design and evaluation of selfnano emulsifying drug delivery system of flutamide. J Young Pharm 2011:3(1):4-8.

19. Emami J, Tajeddin M, Ahmadi F. Preparation and in vitro evaluation of sustained-release matrix tablets of flutamide using synthetic and naturally occurring polymers. Iran J Pharm Res 2008;7(4):247-57.

20. Elkhodairy KA, Hassan MA, Afifi SA. Formulation and optimization of orodispersible tablets of flutamide. Saudi Pharm J 2014;22(1):53-61.

21. Velcheti V, Karnik S, Bardot SF, Prakash O. Pathogenesis of prostate cancer: Lessons from basic research. Ochsner J 2008;8(4):213-8.

22. Yeole BB. Trends in the prostate cancer incidence in India. Asian Pac J Cancer Prev 2008;9(1):141-4.

23. Benedettini E, Nguyen P, Loda M. The pathogenesis of prostate cancer: From molecular to metabolic alterations. Diagn Histopathol (Oxf) 2008;14(5):195-201.

24. Mahmoud AM, Al-Alem U, Dabbous F, Ali MM, Batai K, et al. Zinc intake and risk of prostate cancer: Case-control study and meta-analysis. PLoS One 2016;11(11):e0165956.

25. Sandeep S, Raguvaran R, Rajkumar K. Factors influencing prostate cancer. Int J Pharm Pharm Sci 2014;6(6):33-5.

26. Yallapu MM, Dobberpuhl MR, Maher DM, Jaggi M, Chauhan SC. Design of curcumin loaded cellulose nanoparticles for prostate cancer. Curr Drug Metab 2012;13(1):120-8.

27. Chandratre SS, Dash AK. Multifunctional nanoparticles for prostate cancer therapy. AAPS Pharm Sci Tech 2014;16(1):98-107.

28. Pouton CW. Lipid formulations for oral administration of drugs: Nonemulsifying, self-emulsifying and self-micro emulsifying drug delivery systems. Eur J Pharm Sci 2000;11:93-8.

29. Suciati T, Aliyandi A, Satrialdi. Development of transdermal nanoemulsion formulation for simultaneous delivery of protein vaccine and artin-M adjuvant. Int J Pharm Pharm Sci 2014;6(6):536-46.

30. Rachmawatil H, Yee CW, Rahma A. Formulation of tablet containing curcumin nanoemulsion. Int J Pharm Pharm Sci 2014;6(3):115-20. 